\title{
Is Dental Implant Placement Compatible in Patients Treated with Bisphosphonates? A Literature Review
}

ISSN: 2637-7764

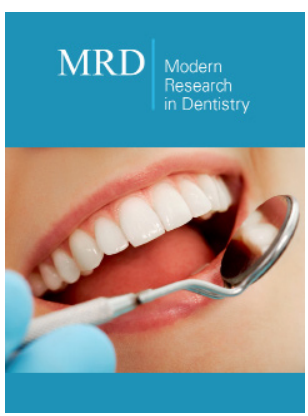

*Corresponding author: Cristina Meniz García, Department of Dental Clinic Specialties, Faculty of Dentistry, Plaza Ramón y Cajal S/N, 28040, Complutense University of Madrid, Spain

Email: cmenizga@ucm.es

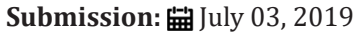

Published: 柴July 19, 2019

Volume 4 - Issue 2

How to cite this article: Iglesias Velázquez 0, Pérez González F, Sánchez Labrador L, Meniz García C, López Quiles J. Is Dental Implant Placement Compatible in Patients Treated with Bisphosphonates? A Literature Review. Mod Res Dent. 4(2). MRD.000585.2019.

DOI: 10.31031/MRD.2019.04.000585

Copyright@ Meniz García C, This article is distributed under the terms of the Creative Commons Attribution 4.0 International License, which permits unrestricted use and redistribution provided that the original author and source are credited.

\section{Meniz García $\mathrm{C}^{1 *}$, Iglesias Velázquez $\mathrm{O}^{2}$, Pérez González $\mathrm{F}^{3}$, Sánchez Labrador} $\mathrm{L}^{3}$ and López Quiles $\mathrm{J}^{1}$

${ }^{1}$ Professor at Department of Dental Clinical Specialties, Faculty of Dentistry, Complutense University of Madrid, Spain

${ }^{2}$ Graduate, Faculty of Dentistry, Complutense University of Madrid, Spain

${ }^{3}$ Department of Dental Clinical Specialties, Faculty of Dentistry, Complutense University of Madrid, Spain

\begin{abstract}
Background: Bisphosphonates (BP) are a group of drugs used in treatment and prevention of alterated turn-over of bone diseases. Since 2003, it is known That BP are able to develop osteonecrosis of the jaw (BRONJ: Bisphosphonate-Related Osteonecrosis of the Jaw) when they are associated to any surgical trauma, even dental implant therapy.
\end{abstract}

Materials and Methods: Comprehensive research of scientific articles from ten years ago to our days in Medline (Pubmed).

Results and Discussion: 1090 dental implants placed patients treated with BP and 3472 dental implants placed in healthy patients had successfully rates of $97.6 \%$ and $97.9 \%$, respectively. Therefore, implant placement in patients in treatment with BP does not reduce implant success rates. Besides, BRONJ appears before with intravenous vs oral administration of BP.

Conclusion: Further well-designed long-term studies are necessary to evaluate correctly the relationship between BP and dental implants.

Keywords: Dental implant; Osteonecrosis; Bisphosphonate; Bisphosphonate-related osteonecrosis of the jaw

Abbreviations: BP: Bisphosphonates; BRONJ: Bisphosphonate Related Osteonecrosis of the Jaw; CTX: C Terminal Telopeptide, TCT; AAOMS: The American Association of Oral and Maxillofacial Surgeons

\section{Introduction}

Bisphosphonates (BP) are a group of drugs used for prevention and treatment of diseases involving bone resorption (malignant tumors such as multiple myeloma, malignant hypercalcemia and other tumors with bone metastases as breast or prostate cancer) and metabolic diseases that affect bones (osteopenia and osteoporosis, present in about 200 million people worldwide). This group of drugs, being the first line therapy in diseases such as osteoporosis, where bone quality and quantity may be affected, are especially important in oral surgery and implantology [1]. These drugs are analogs of pyrophosphate molecule, and cause inhibition of bone reabsorption due to their affinity to calcium phosphate in solid phase, which allow them to bind to bone hydroxyapatite [2,3]. BPs have two forms of administration: intravenous, producing its initial effects after a few days and oral (either daily, weekly, monthly or quarterly), beginning after a few weeks because they are more difficult to be absorbed [3]. In 2003 Marx [4] warned out dental community after noticing the occurrence of osteonecrosis associated with the intake of these drugs. Many studies suggest development of osteonecrosis depends on several factors, such as the total dose, the duration and the way of the drug administration $[1,5,6]$. Osteonecrosis is defined as exposed 
bone in the maxillofacial region for more than eight weeks, in patients treated with BPs without story of head or neck radiation, previously subjected to a dental surgery, for example an exodontia or dental implant placement. Dental implants today have become the most efficient alternative to rehabilitate missing teeth, having better results than conventional fixed and removable prosthesis. Nonetheless, the information about association between dental implants and osteonecrosis is scarce [7].

Although the mechanism of beginning and progression of osteonecrosis associated with the use of BP is unclear, there are several hypotheses suggesting that these drugs inhibit the activity of osteoclasts, suppressing bone remodeling. It seems to be related to the surgical trauma, bad hygiene or periodontal and peri implant status $[5,8,9]$. According to American Dental Association Council on Scientific Affairs in 2006, the incidence of osteonecrosis of the jaw associated with the use of oral BP is estimated at 0.7 cases per 100,000 persons / year of exposure [10]. The implant success depends on osseointegration, defined as the direct, structural and functional connection between the live, ordered bone and the surface of an implant under functional loading. Patients being treated with BP have an affected bone remodeling process, because of the inhibition of osteoclasts' action. This prevents the first phase, which allows osteogenesis to occur, so a succesfully osseointegration is not expected to occur and implant prognosis is also questionable [5,8]. However, in the literature there are several studies showing beneficial effects of oral BP in osseointegration and bone healing around implants [5]. The aim of this review is to evaluate the behaviour of dental implants in patients treated with $\mathrm{BP}$, and the protocol to follow with implants in patients employing BP as medication, or in patients who develop complications associated with its administration.

\section{Material and Methods}

A literature search was conducted in different digital databases like Medline (Pubmed) and Cochrane using keywords ("implant dental" AND "osteonecrosis" AND "dental implant failure") AND ("bisphosphonate" AND "bisphosphonate-related osteonecrosis of the jaw ") until May 2019. The search was carried out by a single subject (OI). According to the keywords and search strategy, 5971 results were obtained. The search was limited to journal articles in the last 15 years and the field of Medicine, Dentistry and Pharmacology, limiting the search to 2618 publications. The selection of studies was limited further by focusing on the field of Oral and Maxillofacial Surgery, publications with highest level of scientific evidence and written mainly in English. Articles about one case, case series and follow-up less of six-months were excluded. Thus 39 publications were included.

\section{Result and Discussion}

The high incidence in the Spanish society of diseases such as osteoporosis, one of the oldest in the world (affecting about 35\% of women over seventy years) is a public health problem. This disease, where bone quality and quantity are affected, seems to be a contraindication for dental implants. If these patients are taking oral or intravenous BPs, the problem seems to be even greater ${ }^{10}$. Dental implants placement in patients treated with BP is a controversial option. For some authors, these drugs represent a contraindication to perform any oral surgery [4]. In fact, there is a consensus to contraindicate the use of implants in cancer patients treated with intravenous BP due to high risk of BRONJ, in order to prevent the development of complications such as those mentioned by Marx [4] (pain, infection and necrosis) [5]. However, several studies do not share this theory, by inducing osteoporosis in a group of rabbits (giving them zoledronate) they concluded BPs could reverse the effects of osteoporosis and facilitate osseointegration, and even could also be an effective strategy to promote osseointegration of implants in patients with metabolic bone diseases $[11,12]$.

Ata Ali et al. [13] published a systematic review that analises the impact of BP for implant success. Their analysis included fourteen studies: Ten retrospective, two prospective and two case series; even though it was focused mainly on eight studies. They evaluated a total of 386 cases of patients with BP and 902 healthy subjects where 1090 and 3472 implants were placed, respectively. They obtained a success rate of $97.61 \%$ in the group with BP and $97.81 \%$ in the healthy group, so there is enough evidence to ensure that BP have no negative effect on implant survival [13]. Jeffcoat [14] in a study of 25 patients with oral BP achieved similar success rates to the healthy group [14] and Bell \& Bellet [15] evaluating 100 implants in the group with oral BP reached similar results to healthy group [15]. In this way, these studies match a study in postmenopausal women treated with oral BP, which recorded a success rate of $100 \%$ implants with one year of follow-up [16].

The systematic review of Madrid \& Sanz [6] analized four studies: one prospective controlled nonrandomized, which compared patients with and without BP up to 36 months after implant placement and without patients developing osteonecrosis, with a satisfactory implant's response. The other three studies were retrospective, and had similar results, because all patients taking oral BP did not develop osteonecrosis and reported success rates of dental implants close to $100 \%$. Anyway, these results should be interpreted carefully because the sample was limited and in all four studies the duration of BP exposure regarding implants was quite short (less than four years). The systematic review concluded that risk of osteonecrosis is minimal when the administration of BP is less than five years [5], but the use of BP presents similar results to healthy patients. However, there are also different cases in the literature that showed implant failures $[17,18]$. Even they say BP oral intake increases the probability of develop osteonecrosis 2.69 times [19]. According to several studies, the appearance of osteonecrosis is related to the type of BP, dose, potency or way of administration. It has also been found that may be associated with poor hygiene, smoke or systemic condition $[1,3,4,6]$. Regarding the way of administration, osteonecrosis develops earlier in patients taking intravenous versus oral administration. Marx [11] reported a success rate of $97,5 \%$ in dental Implants placed in patients treated with oral BP [20], Ruggiero 89\% [20], Mavrokokki 77\% [21] or Abu-Id 93\% [22]. The systematic review of Madrid \& Sanz showed a 
success rate of $100 \%$ in patients treated with oral BP [5]. However, although osteonecrosis tends to appear faster in patients receiving intravenous therapy, it does not exist a significant difference in the form of administration, the dose and the development of osteonecrosis [4]. Recently, a systematic review of Gelazius et al. [9] reported success rates of $100 \%$ in most studies evaluated, finding the lowest success rates in patients treated with intravenous BP $[8,23]$.

One of the absolute contraindications in oral and maxillofacial surgery is implants placement in patients with intravenous BP for cancer treatment. Despite this, a study in Japan on six patients with implants reported a single case of osteonecrosis. Although the sample size is not significant, they considered that implants did not represent a risk factor in these patients [24]. Another study in cancer patients who developed osteonecrosis in relation to implants concluded that, despite being Osseo integrated, the disease could be developed in the long term and not only in relation with the surgical act [25]. This statement is consistent in Giovannacci et al. study, which reported some cases of osteonecrosis both in patients who undergo implant placement surgery and in those who were already Osseo integrated. In addition, the study concluded that the incidence of this pathology is greater in the posterior regions of the jaws and in patients who have been using BP for more than three years, increasing the risk even more if these drugs are administered intravenously or the patient are simultaneous being treated with corticosteroids [26].

Periimplantitis is one of the most frequent complications in Implant treatments $[27,28]$. In a study of 316 patients where the $39 \%$ of implants ( $n=117)$ showed any signs of periimplantitis (inflammation and bone loss). Of 117 implants, 111 were placed before initiating therapy with $\mathrm{BP}$, so the use of these drugs is a risk factor for developing periimplantitis [29]. Prevention in these patients must be vital, consisting in regular visits to the dentist making evaluations of the periodontal status and informing patients about the negative effects of this type of drugs in the oral cavity, spontaneously or in relation to dental activity. One of the practices that is carried out is the "drug-holiday" or BP break. Some authors accept this theory based on the blood determination of CTX levels (C-terminal telopeptide, TCT). It is a specific marker that is released in the serum by the action of osteoclasts and indicates the risk of developing osteonecrosis. When the values are above $150 \mathrm{pg} / \mathrm{ml}$, the risk of developing osteonecrosis is minimal. On the other hand, when these values are lower, it is recommended to suspend the BP three months before placing the implants and waiting another three months to restore the use, in order to achieve a correct healing. However, the AAOMS (The American Association of Oral and Maxillofacial Surgeons) warn that stopping medication does not reduce the risk of osteonecrosis $[3,6,15]$. In contrast, the study by Koka et al. comparing a group with implants and BP and another group without medication, does not find a statistically significant difference, so it would not be necessary to perform the "drugholiday" [29], coinciding with other studies where the difference was not significant either [30]. Marx [4] argues that any form of prevention of osteonecrosis associated with BP is very complicated and the works of Hewitt et al. or Farah et al., recommend performing any surgical treatment before starting pharmacological therapy [31]. In other hand, alternative therapies involving different materials or biomolecules has been also investigated like the possibility of using substitutes for BP locally, with growth hormone or calcium and phosphate supplements, noticing an acceleration in bone formation and improvement in osseointegration [32-36].

On the other hand, the possibility of modifying the surface of the implants with calcium and phosphate molecules or with BP has been studied. The meta-analysis of Alghamdi et al. [37] showed an improvement in osseointegration when the implants surface is treated with these compounds. However, more studies are needed to confirm these hypotheses [36-38]. When osteonecrosis is developed, there are different approaches: an aggressive posture, combining the treatment of kidnappings with explantation $[4,25]$; or a more conservative approach consisting in stopping administering BP and prescribing an antibiotic therapy for several months. If after a long period there are no signs of improvement, explantantion is carried out. Thus, a study in 27 patients who developed osteonecrosis associated with the implants, received a dose of amoxicillin ( $n=7)$ and doxycycline $(n=20)$.

Patients who had taken oral BP showed a more satisfactory response to those who received intravenous BP. Besides, those treated with doxycycline had a better recovery (due to the higher bone affinity of this antibiotic to the bone) compared to the amoxicillin group [1]. Special care should be taken with new generation drugs such as Denosumab (Prolia ${ }^{\circledR}$, Xgeva ${ }^{\circledR}[$ Amgen Technology Ireland (ADL) Dublin, Ireland]). These are monoclonal antibodies used, among other things, for arthritis and osteoporosis. It has been seen that they produce a reduction in bone resorption close to $80 \%$ in the first months [3]. Even so, both the FDA (Food and Drug Administration), and the AEMPS (Spanish Agency for Medicines and Health Products) and different authors, warn about the risk of developing osteonecrosis associated with these drugs [39]. The potential risk of developing osteonecrosis in patients under treatment with BP should be explained, before or after the placement of the implants. The AAOMS recommend a protocol for dental implants placement:

a. If the patient has taken oral BP for less than 3 years and does not have any other risk factor: treatment plan should not be changed, but it is recommended to make an adequate informed consent, and make an interconsultation with the doctor in order to inform and allow to change the medication if it is necessary.

b. If the patient has taken oral BP for less than 3 years but also has any risk factor (e.g. taking corticosteroids): it is recommend to contact the doctor, to stop the treatment if it is possible, from 3 months before implant placement up to 3 months later, to get an adequate bone remodeling.

c. If the patient has taken BP for more than 3 years, with or without any concomitant therapy: an interconsultation should be performed to assess the possibility of stopping the treatment from 3 months before to 3 months later of implants placement. 
d. If the patient takes intravenous BP, dental implants placement is considered an absolute contraindication in cancer patients being treated with zoledronate and pamidronate.

\section{Conclusion}

The success rate of implants in patients with oral BP is very high, so it does not seem to be a risk factor. However, long-term studies with a representative sample are necessary to evaluate the relationship of BP and implants. In addition, to assess the real behaviour between BP and dental implants, studies criteria must be unified taking into account the form of administration, the duration of the treatment, the cessation or not of the drug, relative aspects to dental implant placement or individual characteristics of patients. The professional must advise the patient of the adverse effects of the BP in relation to the surgical intervention and perform an adequate informed consent.

\section{References}

1. Lazorovic T, Yahalom R, Taicher S, Schwartz Arad D, Peleg O, et al. (2010) Bisphosphonate-related osteonecrosis of the jaw associated with dental implants. J Oral Maxilofac Surg 68(4): 790-796.

2. Rigo MA, Díez de Pinos JL, Creus MT, Jané CC, Sala JR (2000) Clinical review of diphosphonate use. Farm Hosp 24(2): 74-82.

3. Otomo Corgel J (2012) Osteoporosis and osteopenia: implications for periodontal and implant therapy. Periodontol 2000. 59(1): 111-139.

4. Marx RE, Sawatari Y, Fortin M, Broumand V (2005) Bisphosphonate induced exposed bone (osteonecrosis/osteopetrosis) of the jaws: Risk factors, recognition, prevention, and treatment. J Oral Maxillofac Surg 63(11): 1567-1575.

5. Holzinger D, Seeman R, Matoni N, Ewers R, Millesi W, et al. (2014) Effect of dental implants on bisphosphonate-related osteonecrosis of the jaws. J Oral Maxillofac Surg 72(10): 1937.e1-8.

6. Madrid C, Sanz M (2009) What impact do systemically administrated bisphosphonates have on oral implant therapy? A systematic review. Clin Oral Impl Res 20(4): 87-95.

7. Qamheya AHA, Yeniyol S, Arisan V (2016) Bisphosphonate-Related Osteonecrosis of the Jaw and Dental Implants. J Istanb Univ Fac Dent 50(1): 59-64.

8. Kwon T, Lee C, Park J, Choi S, Rijal G, et al. (2014) Osteonecrosis associated with dental implants in patients undergoing bisphosphonate treatment. Clin Oral Impl Res 25(5): 632-640.

9. Gelazius R, Poskevicius L, Sakavicius D, Grimuta V, Juodzbalys G (2018) Dental implant placement in patients on bisphosphonate therapy: a systematic review. J Oral Maxillofac Res 9(3): e2.

10. Edwards BJ, Hellstein JW, Jacobsen PL, Kaltman S, Mariotti A, et al. (2008) Updated recommendations for managing the care of patients receiving oral bisphosphonate therapy: an advisory statement from the American Dental Association Council on Scientific Affairs. J Am Dent Assoc 139(12): 1674-1677.

11. Siebert T, Jurkovic R, Statelova D, Strecha J (2015) Immediate implant placement in a patient with osteoporosis undergoing bisphosphonate therapy: 1-year preliminary prospective study. J Oral Implantol 41(Spec No): 360-365.

12. Mengchun Q, Jing H, Jianyuan L, Wei D, Xiaojie F, et al. (2012) Effect of zoledronate acid treatment on osseointegration and fixation of implants in autologous iliac bone grafts in ovariectomized rabbits. Bone 50(1): 119-127.
13. Ata Ali J, Ata Ali F, Oltra DP, Moreno PG (2016) What is the impact of bisphosphonate therapy upon dental implant survival? A systematic review and meta-analysis. Clin Oral Impl Res 27(2): e38-46.

14. Jeffcoat MK (2006) Safety of oral bisphosphonates: controlled studies on alveolar bone. Int J Oral Maxillofac Implants 21(3): 349-353.

15. Bell BM, Bell RE (2008) Oral bisphosphonates and dental implants: a retrospective study. J Oral Maxillofac Surg 66(5): 1022-1024.

16. Martínez García ML (2013) Evaluación del comportamiento de implantes osteointegrados en mujeres con osteoporosis en tratamiento con bisfosfonatos orales. Variabilidad en el tiempo y relación pronóstica del telopéptido carboxiterminal CTX Crosslaps [post doc]. Complutense University, Madrid, Spain, p. 192.

17. Torres J, Tamimi F, Garcia I, Cebrian JL, Lopez Cabarcos E, et al. (2008) Management of atrophic maxilla in severe osteoporosis treated with bisphosphonates: a case report. Oral Surg Oral Med Oral Pathol Oral Radiol Endod 106(5): 668-672.

18. De Freitas NR, Lima LB, de Moura MB, Veloso Guedes CC, Simamoto Júnior PC, et al. (2016) Bisphosphonate treatment and dental implants: A systematic review. Med Oral Patol Oral Cir Bucal 21(5): e644-651.

19. Yip JK, Borrell LN, Cho SC, Francisco H, Tarnow DP (2012) Association between oral bisphosphonate use and dental implant failure among middle-aged women. J Clin Periodontol 39(4): 408-414.

20. Ruggiero SL, Mehrotra B, Rosenberg TJ, Engroff SL (2004) Osteonecrosis of the jaws associated with the use of bisphosphonates: A review of 63 cases. J Oral Maxillofac Surg 62(5): 527-534.

21. Mavrokokki T, Cheng A, Stein B, Gross A (2007) Nature and frequency of bisphosphonate-associated osteonecrosis of the jaws in Australia. J Oral Maxillofac Surg 65(3): 415-423.

22. Abu Id MH, Warnke PH, Gottschalk J, Springer I, Wiltfang J, et al. (2008) "Bis-phossy jaws"-High and low risk factors for bisphosphonate-induced osteonecrosis of the jaw. J Craniomaxillofac Surg 36(2): 95-103.

23. Granate Marques A, Polis Yanes C, Seminario Amez M, Jané Salas E, LópezLópez J (2019) Medication-related osteonecrosis of the jaw associated with implant and regenerative treatments: Systematic review. Med Oral Patol Oral Cir Bucal 24(2): e195-203.

24. Matsuo A, Hamada H, Takahashi H, Okamoto A, Kaise H, et al. (2016) Evaluation of dental implants as a risk factor for the development of bisphosphonate-related osteonecrosis of the jaw in breast cancer patients. Odontology 104(3): 363-371.

25. Figuero E, Graziani F, Sanz I, Herrera D, Sanz M (2014) Management of peri-implant mucosisitis and peri-implantitis. Periodontol 2000 66(1): 255-273.

26. Giovannacci I, Meleti M, Manfredi M, Mortellaro C, Greco Lucchina A, et al. (2016) Medication-related osteonecrosis of the jaw around dental implants: implant surgery-triggered or implant presence-triggered osteonecrosis? J Craniofac Surg 27(3): 697-701.

27. Corcuera Flores JR, Alonso Domínguez AM, Serrera Figallo MA, Torres Lagares D, Castellanos Cosano L, et al. (2016) Relationship between osteoporosis and marginal bone loss in osseointegrated implants: A 2-year retrospective study. J Periodontol 87(1): 14-20.

28. Troeltzch M, Cagna D, Stähler P, Probst F, Kaeppler G, et al. (2016) Clinical features of peri-implant medication-related osteonecrosis of the jaw: is there an association to peri-implantitis? J Craniomaxillofac Surg 44(12): 1945-1951.

29. Koka S, Babu NM, Norell A (2010) Survival of dental implants in postmenopausal bisphosphonate users. J Prosthodont Res 54(3): 108-111.

30. Memon S, Weltman RL, Katancik JA. (2012) Oral bisphosphonates: Early endosseous dental implant success and crestal bone changes. A retrospective study. Int J Oral Maxillofac Implants 27(5): 1216-1222. 
31. Fernandez Ayora A, Herion F, Rompen E, Reginster JY, Magremanne M, et al. (2015) Dramatic osteonecrosis of the jaw associated with oral bisphosphonates, periodontitis and dental implant removal. J Clin Periodontol 42(2): 190-195.

32. Martín Monje EM (2008) Osteoporosis experimental: efecto de la administración local de hormona del crecimiento en el tejido periimplantario [post doc]. Complutense University, Madrid, Spain, p. 534.

33. Nepal M, Li L, Bae TS, Kim BI, Soh Y (2014) Evaluation of osseointegration around tibial implants in rats by ibandronate-treated nanotubular ti32nb-5zr alloy. Biomol Ther 22(6): 563-569.

34. Takahiro T, Takehiro W, Hiroshi N, Yasuhiro T, Suguru K, et al. (2016) Effect of a dietary supplement on peri-implant bone strength in a rat model on osteoporosis. J Prosthodont Res 60(2): 131-137.

35. Kellesarian SV, Subhi ALHarthi S, Saleh Binshabaib M, Javed F (2017) Effect of local zoledronate delivery on osseointegration: a systematic review of preclinical studies. Acta Odontol Scand 75(7): 530-541.
36. Thirunavukarasu A, Pinto HG, Seymour KG (2015) Bisphosphonate and implant dentistry-Is it safe? Prim Dent J 4(3):30-33.

37. Alghamdi HS, Junker R, Bronkhorst EM, Jansen JA (2012) Bone Regeneration related to calcium phosphate-coated implants in osteoporotic animal models: A meta-analysis. Tissue Eng Part B Rev 18(5): 383-395.

38. Aspenberg P (2006) Osteonecrosis of the jaw: What do bisphosphonates do? Expert Opin Drug Saf 5(6): 743-745.

39. Javed F, Almas K (2010) Osseointegration of dental implants in patients undergoing bisphosphonate treatment: a literature review. J Periodontol 81(4): 479-484. 